\title{
Article \\ A Projected Forward-Backward Algorithm for Constrained Minimization with Applications to Image Inpainting
}

\author{
Suthep Suantai ${ }^{1}$, Kunrada Kankam ${ }^{2}$ and Prasit Cholamjiak ${ }^{2, *}$ \\ 1 Data Science Research Center, Department of Mathematics, Faculty of Science, Chiang Mai University, \\ Chiang Mai 50200, Thailand; suthep.s@cmu.ac.th \\ 2 School of Science, University of Phayao, Phayao 56000, Thailand; kunradazzz@gmail.com \\ * Correspondence: prasitch2008@yahoo.com or prasit.ch@up.ac.th
}

check for updates

Citation: Suantai, S.; Kankam, K.; Cholamjiak, P. A Projected ForwardBackward Algorithm for Constrained Minimization with Applications to Image Inpainting. Mathematics 2021, 9, 890. https://doi.org/10.3390/ math9080890

Academic Editor: Raimondas Ciegis

Received: 26 March 2021

Accepted: 12 April 2021

Published: 16 April 2021

Publisher's Note: MDPI stays neutral with regard to jurisdictional claims in published maps and institutional affiliations.

Copyright: (c) 2021 by the authors. Licensee MDPI, Basel, Switzerland. This article is an open access article distributed under the terms and conditions of the Creative Commons Attribution (CC BY) license (https:/ / creativecommons.org/licenses/by/ $4.0 /)$.

\begin{abstract}
In this research, we study the convex minimization problem in the form of the sum of two proper, lower-semicontinuous, and convex functions. We introduce a new projected forward-backward algorithm using linesearch and inertial techniques. We then establish a weak convergence theorem under mild conditions. It is known that image processing such as inpainting problems can be modeled as the constrained minimization problem of the sum of convex functions. In this connection, we aim to apply the suggested method for solving image inpainting. We also give some comparisons to other methods in the literature. It is shown that the proposed algorithm outperforms others in terms of iterations. Finally, we give an analysis on parameters that are assumed in our hypothesis.
\end{abstract}

Keywords: convex minimization; image inpainting; inertial techniques; weak convergence

\section{Introduction}

Let $H$ be a real Hilbert space. The unconstrained minimization problem of the sum of two convex functions is modeled as the following form:

$$
\min _{u \in H} f(u)+g(u)
$$

where $f$ and $g: H \rightarrow \mathbb{R} \cup\{+\infty\}$ are proper, lower semi-continuous, and convex functions. If $f$ is differentiable on $H$, we know that problem (1) can be described by the fixed point equation, that is,

$$
u=\operatorname{prox}_{\alpha g}(u-\alpha \nabla f(u))
$$

where $\alpha>0$ and prox $_{g}$ is the proximal operator of $g$, i.e., $\operatorname{prox}_{g}=(I d+\partial g)^{-1}$ where $I d$ is the identity operator in $H$ and $\partial g$ is the subdifferential of $g$. Therefore, the forwardbackward algorithm was defined in the following manner:

$$
u^{k+1}=\underbrace{\operatorname{prox}_{\alpha_{k} g}}_{\text {backward step }} \underbrace{\left(I d-\alpha_{k} \nabla f\right)}_{\text {forward step }}\left(u^{k}\right), k \geq 0
$$

where $\alpha_{k}>0$. Some works that relate to the forward-backward method for convex optimization problems can be investigated in [1-6]. This method covers the gradient method [7-9] and the proximal algorithm [10-12]. Combettes and Wajs [13] introduced the following relaxed forward-backward method.

Cruz and Nghia [14] suggested the forward-backward method using linesearch approach, which does not need the Lipschitz constant in implementation.

It was shown that $\left(u^{k}\right)$ converges weakly to a minimizer of $f+g$.

Now, inspired by Cruz and Nghia [14], we suggest a new projected forward-backward algorithm for solving the constrained convex minimization problem, which is modeled as follows: 


$$
\min _{u \in \Omega} f(u)+g(u),
$$

where $\Omega$ is a nonempty closed convex subset of $H, f$ and $g$ are convex functions on $H$ that $f$ is differentiable on $H$. We denote by $S_{*}$ the solution set of (4).

By the way, to obtain a nice convergence rate, Polyak [15] introduced the heavy ball method for solving smooth convex minimization problem. In case $g=0$, Nesterov [16] modified the heavy ball method as follows.

In this work, motivated by Algorithm 1 [13], Algorithm 2 [14], Algorithm 3 [16] and Algorithm 4 [17], we are interested to design a new projected forward-backward algorithm for solving the constrained convex minimization problem (4) and establishing the convergence theorem. We also apply our method to solve image inpainting and provide some comparisons and numerical results. Finally, we show the effects of each parameters in the proposed algorithm.

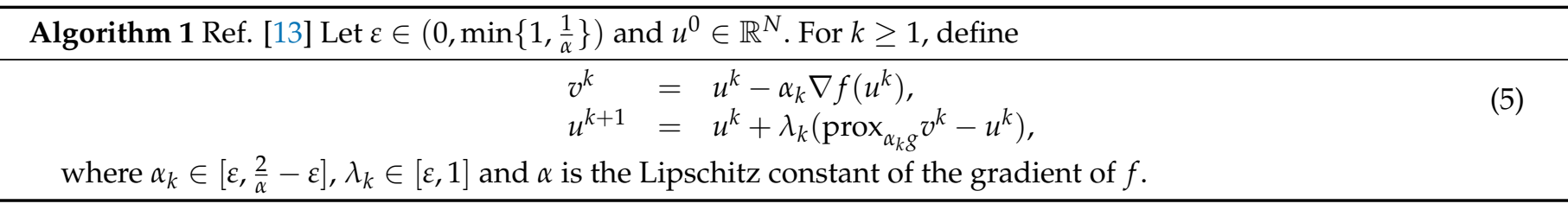

Algorithm 2 Let $\sigma>0, \theta \in(0,1)$ and $\delta \in\left(0, \frac{1}{2}\right)$. Let $u^{0} \in \operatorname{dom} g$. For $k \geq 1$, define

$$
u^{k+1}=\operatorname{prox}_{\alpha_{k} g}\left(u^{k}-\alpha_{k} \nabla f\left(u^{k}\right)\right),
$$

where $\alpha_{k}=\sigma \theta^{m_{k}}$ and $m_{k}$ is the smallest nonnegative integer satisfying

$$
\alpha_{k}\left\|\nabla f\left(u^{k+1}\right)-\nabla f\left(u^{k}\right)\right\| \leq \delta\left\|u^{k+1}-u^{k}\right\| .
$$

Algorithm 3 Let $u^{0}, u^{1} \in \mathbb{R}^{N}$ and $\theta_{k} \in[0,1)$. For $k \geq 1$, define

$$
\begin{aligned}
v^{k} & =u^{k}+\theta_{k}\left(u^{k}-u^{k-1}\right), \\
u^{k+1} & =v^{k}-\alpha_{k} \nabla f\left(v^{k}\right),
\end{aligned}
$$

where $\alpha_{k}>0$. The term $\theta_{k}\left(u^{k}-u^{k-1}\right)$ is called inertial term.

In 2003, Moudafi and Oliny [17] suggested the inertial forward-backward splitting as follows:

Algorithm 4 Let $u^{0}, u^{1} \in \mathbb{R}^{N}$ and $\alpha_{k} \in\left[0, \frac{2}{L}-\varepsilon\right]$. For $k \geq 1$, define

$$
\begin{aligned}
v^{k} & =u^{k}+\theta_{k}\left(u^{k}-u^{k-1}\right), \\
u^{k+1} & =\operatorname{prox}_{\alpha_{k g} g}\left(v^{k}-\alpha_{k} \nabla f\left(u^{k}\right)\right),
\end{aligned}
$$

where $\theta_{k} \in[0,1)$. Many works explored that algorithms involving inertial term have a nice rate of convergence [3,18-20]. The complexity of some variants of the forward-backward algorithms can be found in the work of Cruz and Nghia [14].

This paper is organized as follows: In Section 2, we recall some preliminaries and mathemetical tools. In Section 3, we prove the weak convergence theorem of the proposed method. In Section 4, we provide numerical experiments in image inpainting to valid the convergence theorem and, finally, in Section 5, we give conclusions of this paper. 


\section{Preliminaries}

Let us review some important definitions and lemmas for proving the convergence theorem. Let $H$ be a real Hilbert space with inner product $\langle\cdot, \cdot\rangle$ and norm $\|\cdot\|$. Let $h: H \rightarrow \overline{\mathbb{R}}$ be a proper, lower semicontinuous (l.s.c.), and convex function. The domain of $h$ is defined by dom $h:=\{u \in H \mid h(u)<+\infty\}$. For any $u \in H$, we know that the orthogonal projection of $u$ onto a nonempty, closed and convex subset $C$ of $H$ is defined by

$$
P_{C} u:=\underset{v \in C}{\operatorname{argmin}}\|u-v\|^{2} \text {. }
$$

Lemma 1 ([21]). Let $C$ be a nonempty, closed and convex subset of a real Hilbert space H. Then, for any $u \in H$, we have

(i) $\left\langle u-P_{C} u, a-P_{C} u\right\rangle \leq 0$ for all $a \in C$;

(ii) $\left\|P_{C} u-P_{C} v\right\|^{2} \leq\left\langle P_{C} u-P_{C} v, u-y\right\rangle$ for all $u, v \in H$;

(iii) $\left\|P_{C} u-a\right\|^{2} \leq\|u-a\|^{2}-\left\|P_{C} u-u\right\|^{2}$ for all $a \in C$.

The directional derivative of $h$ at $u$ in the direction $d$ is

$$
h^{\prime}(u ; d):=\lim _{t \rightarrow 0^{+}} \frac{h(u+t d)-h(u)}{t} .
$$

Definition 1. The subdifferential of $h$ at $u$ is defined by

$$
\partial h(u)=\{a \in H:\langle a, v-u\rangle \leq h(v)-h(u), y \in H\} .
$$

It is known that $\partial h$ is maximal monotone and if $h$ is differentiable, then $\partial h$ is the gradient of $h$ denoted by $\nabla h$. Moreover, $\nabla h$ is monotone, that is, $\langle\nabla h(u)-\nabla h(v), u-v\rangle \geq 0$ for all $u, v \in H$. From (4), we also know that

$$
u_{*} \in \operatorname{argmin}(f+g) \Leftrightarrow u_{*}=\operatorname{prox}_{c g}(I d-c \nabla f)\left(u_{*}\right)
$$

where $c>0$ and prox $_{g}=(I d+\partial g)^{-1}$.

From [14], we know that

$$
\frac{a-\operatorname{prox}_{\alpha g}(a)}{\alpha} \in \partial g\left(\operatorname{prox}_{\alpha g}(a)\right) \text { for all } a \in H, \alpha>0 \text {. }
$$

Lemma 2 ([22]). $\partial h, \operatorname{Gph}(\partial h)=\{(u, a) \in H \times H: a \in \partial h(u)\}$ is demiclosed, i.e., if the sequence $\left(u^{k}, a^{k}\right) \subset \mathrm{Gph}(\partial h)$ satisfies that $\left(u^{k}\right)$ converges weakly to $u$ and $\left(a^{k}\right)$ converges strongly to $a$, then $(u, a) \in \mathrm{Gph}(\partial h)$.

Lemma 3. Let $\left(a^{k}\right),\left(b^{k}\right)$ and $\left(r_{k}\right)$ be real positive sequences such that

$$
\begin{array}{r}
a^{k+1} \leq\left(1+r_{k}\right) a^{k}+b^{k}, k \in \mathbb{N} . \\
\text { If } \sum_{k=1}^{\infty} r_{k}<+\infty \text { and } \sum_{k=1}^{\infty} b^{k}<+\infty \text {, then } \lim _{k \rightarrow+\infty} a^{k} \text { exists. }
\end{array}
$$

Lemma 4 ([23]). Let $\left(a^{k}\right)$ and $\left(\theta_{k}\right)$ be real positive sequences such that

$$
a^{k+1} \leq\left(1-\theta_{k}\right) a^{k}+\theta_{k} a^{k-1}, k \in \mathbb{N}
$$

Then, $a^{k+1} \leq K \cdot \prod_{j=1}^{k}\left(1+2 \theta_{j}\right)$, where $K=\max \left\{a_{1}, a_{2}\right\}$. Moreover, if $\sum_{k=1}^{\infty} \theta_{k}<+\infty$, then $\left(a^{k}\right)$ is bounded. 
Definition 2. Let $S$ be a nonempty subset of $H$. A sequence $\left(u^{k}\right)$ in $H$ is said to be quasiFejér convergent to $S$ if and only if for all $u \in S$ there exists a positive sequence $\left(\varepsilon_{k}\right)$ such that $\sum_{k=0}^{\infty} \varepsilon_{k}<+\infty$ and $\left\|u^{k+1}-u\right\|^{2} \leq\left\|u^{k}-u\right\|^{2}+\varepsilon_{k}$ for all $k \in \mathbb{N}$. When $\left(\varepsilon_{k}\right)$ is a null sequence, we say that $\left(u^{k}\right)$ is Fejér convergent to $S$.

Lemma 5 ([21,24]). If $\left(u^{k}\right)$ is quasi-Fejér convergent to $S$, then we have:

(i) $\left(u^{k}\right)$ is bounded.

(ii) If all weak accumulation points of $\left(u^{k}\right)$ is in $S$, then $\left(u^{k}\right)$ weakly converges to a point in $S$.

\section{Results}

In this section, we suggest a new projected forward-backward algorithm and establish the weak convergence. The following conditions are assumed:

(A1) $f, g: H \rightarrow \mathbb{R} \cup\{+\infty\}$ are proper, l.s.c, convex functions on $H$ that $f$ is differentiable on $H$.

(A2) $\nabla f$ is uniformly continuous on bounded subsets of $H$ and is bounded on any bounded subset of $H$.

Next, we will prove weak convergence theorem of the proposed algorithm.

Theorem 1. Let $\left(u^{k}\right)$ be defined by Algorithm 5. Suppose $\sum_{k=1}^{\infty} \theta_{k}<+\infty$. Then, $\left(u^{k}\right)$ weakly converges to a point in $S_{*}$.

Algorithm 5 Let $\Omega$ be a nonempty closed convex subset of $H$. Given $\sigma>0$, $\phi \in(0,1), \delta \in\left(0, \frac{1}{2}\right)$ and $\theta_{k} \geq 0$. Let $u^{0}, u^{1} \in H$ and define

$$
w^{k}=u^{k}+\theta_{k}\left(u^{k}-u^{k-1}\right)
$$

and

$$
v^{k}=\operatorname{prox}_{\alpha_{k g}}\left(w^{k}-\alpha_{k} \nabla f\left(w^{k}\right)\right)
$$

where $\alpha_{k}=\sigma \phi^{m_{k}}$ and $m_{k}$ is the smallest nonnegative integer such that

$$
\alpha_{k}\left\|\nabla f\left(v^{k}\right)-\nabla f\left(w^{k}\right)\right\| \leq \delta\left\|v^{k}-w^{k}\right\| .
$$

Set $u^{k+1}$ by

$$
u^{k+1}=P_{\Omega}\left(v^{k}\right), k \geq 0
$$

Proof. Let $u_{*}$ be a solution in $S_{*}$. Thus, we obtain

$$
\begin{aligned}
\left\|u^{k+1}-u_{*}\right\|^{2} & =\left\|P_{\Omega}\left(v^{k}\right)-u_{*}\right\|^{2} \\
& \leq\left\|v^{k}-u_{*}\right\|^{2}-\left\|P_{\Omega}\left(v^{k}\right)-v^{k}\right\|^{2} .
\end{aligned}
$$

By the definition of proximal operator and $v^{k}$, we have

$$
\frac{w^{k}-v^{k}}{\alpha_{k}}-\nabla f\left(w^{k}\right)=\frac{w^{k}-\operatorname{prox}_{\alpha_{k} g}\left(w^{k}-\alpha_{k} \nabla f\left(w^{k}\right)\right)}{\alpha_{k}}-\nabla f\left(w^{k}\right) \in \partial g\left(v^{k}\right) .
$$

By the convexity of $g$, we get

$$
g(u)-g\left(v^{k}\right) \geq\left\langle\frac{w^{k}-v^{k}}{\alpha_{k}}-\nabla f\left(w^{k}\right), u-v^{k}\right\rangle, \forall u \in H .
$$


The convexity of $f$ gives

$$
f(u)-f(y) \geq\langle\nabla f(y), u-y\rangle, \forall u \in H, y \in H .
$$

Using (13) and (14) with any $u \in H$ and $y=w^{k}$, we obtain

$$
\begin{aligned}
g(u)-g\left(v^{k}\right)+f(u)-f\left(w^{k}\right) \geq & \left\langle\frac{w^{k}-v^{k}}{\alpha_{k}}-\nabla f\left(w^{k}\right), u-v^{k}\right\rangle+\left\langle\nabla f\left(w^{k}\right), u-w^{k}\right\rangle \\
= & \frac{1}{\alpha_{k}}\left\langle w^{k}-v^{k}, u-v^{k}\right\rangle+\left\langle\nabla f\left(w^{k}\right), v^{k}-w^{k}\right\rangle \\
= & \frac{1}{\alpha_{k}}\left\langle w^{k}-v^{k}, u-v^{k}\right\rangle+\left\langle\nabla f\left(v^{k}\right), v^{k}-w^{k}\right\rangle \\
& +\left\langle\nabla f\left(w^{k}\right)-\nabla f\left(v^{k}\right), v^{k}-w^{k}\right\rangle \\
\geq & \frac{1}{\alpha_{k}}\left\langle w^{k}-v^{k}, u-v^{k}\right\rangle+\left\langle\nabla f\left(v^{k}\right), v^{k}-w^{k}\right\rangle \\
& -\left\|\nabla f\left(w^{k}\right)-\nabla f\left(v^{k}\right)\right\|\left\|v^{k}-w^{k}\right\| \\
\geq & \frac{1}{\alpha_{k}}\left\langle w^{k}-v^{k}, u-v^{k}\right\rangle+\left\langle\nabla f\left(v^{k}\right), v^{k}-w^{k}\right\rangle \\
& -\frac{\delta}{\alpha_{k}}\left\|w^{k}-v^{k}\right\|^{2} .
\end{aligned}
$$

This yields

$\left\langle w^{k}-v^{k}, v^{k}-u\right\rangle \geq \alpha_{k}\left[f\left(w^{k}\right)-f(u)+g\left(v^{k}\right)-g(u)+\left\langle\nabla f\left(v^{k}\right), v^{k}-w^{k}\right\rangle\right]-\delta\left\|w^{k}-v^{k}\right\|^{2}$.

Since $2\left\langle w^{k}-v^{k}, v^{k}-u\right\rangle=\left\|w^{k}-u\right\|^{2}-\left\|w^{k}-v^{k}\right\|^{2}-\left\|v^{k}-u\right\|^{2}$, it follows that

$$
\begin{aligned}
\left\|w^{k}-u\right\|^{2}-\left\|v^{k}-u\right\|^{2} \geq & 2 \alpha_{k}\left[f\left(w^{k}\right)-f(u)+g\left(v^{k}\right)-g(u)+\left\langle\nabla f\left(v^{k}\right), v^{k}-w^{k}\right\rangle\right] \\
& +(1-2 \delta)\left\|w^{k}-v^{k}\right\|^{2} .
\end{aligned}
$$

Since $f$ is convex, we have $f\left(w^{k}\right)-f\left(v^{k}\right) \geq\left\langle\nabla f\left(v^{k}\right), w^{k}-v^{k}\right\rangle$. This implies that

$$
\begin{aligned}
\left\|w^{k}-u\right\|^{2}-\left\|v^{k}-u\right\|^{2} \geq & 2 \alpha_{k}\left[f\left(w^{k}\right)-f(u)+g\left(v^{k}\right)-g(u)-f\left(w^{k}\right)+f\left(v^{k}\right)\right] \\
& +(1-2 \delta)\left\|w^{k}-v^{k}\right\|^{2} .
\end{aligned}
$$

Setting $u=u_{*}$, we obtain

$$
\begin{aligned}
\left\|v^{k}-u_{*}\right\|^{2} & \leq\left\|w^{k}-u_{*}\right\|^{2}-2 \alpha_{k}\left[(f+g)\left(v^{k}\right)-(f+g)\left(u_{*}\right)\right]-(1-2 \delta)\left\|w^{k}-v^{k}\right\|^{2} \\
& \leq\left\|w^{k}-u_{*}\right\|^{2} .
\end{aligned}
$$

From (12) and (15), we see that

$$
\begin{aligned}
\left\|u^{k+1}-u_{*}\right\|^{2} & \leq\left\|w^{k}-u_{*}\right\|^{2}-\left\|P_{\Omega}\left(v^{k}\right)-v^{k}\right\|^{2} \\
& \leq\left\|w^{k}-u_{*}\right\|^{2} .
\end{aligned}
$$

Hence,

$$
\begin{aligned}
\left\|u^{k+1}-u_{*}\right\| & \leq\left\|w^{k}-u_{*}\right\| \\
& \leq\left\|u^{k}-u_{*}\right\|+\theta_{k}\left\|u^{k}-u^{k-1}\right\| \\
& \leq\left\|u^{k}-u_{*}\right\|+\theta_{k}\left(\left\|u^{k}-u_{*}\right\|+\left\|u^{k-1}-u_{*}\right\|\right) .
\end{aligned}
$$
we have

This shows that $\left\|u^{k+1}-u_{*}\right\| \leq\left(1+\theta_{k}\right)\left\|u^{k}-u_{*}\right\|+\theta_{k}\left\|u^{k-1}-u_{*}\right\|$. By Lemma 4,

$$
\left\|u^{k+1}-u_{*}\right\| \leq K \cdot \prod_{j=1}^{k}\left(1+2 \theta_{j}\right)
$$


where $K=\max \left\{\left\|u^{1}-u_{*}\right\|,\left\|u^{2}-u_{*}\right\|\right\}$. Since $\sum_{k=1}^{\infty} \theta_{k}<+\infty,\left(u^{k}\right)$ is bounded. Thus, $\sum_{k=1}^{\infty} \theta_{k}\left\|u^{k}-u^{k-1}\right\|<+\infty$. By Lemma 3, therefore $\lim _{k \rightarrow \infty}\left\|u^{k}-u_{*}\right\|$ exists.

Next, we consider

$$
\begin{aligned}
\left\|w^{k}-u_{*}\right\|^{2} & =\left\|u^{k}+\theta_{k}\left(u^{k}-u^{k-1}\right)-u_{*}\right\|^{2} \\
& =\left\|u^{k}-u_{*}\right\|^{2}+2 \theta_{k}\left\langle u^{k}-u_{*}, u^{k}-u^{k-1}\right\rangle+\theta_{k}^{2}\left\|u^{k}-u^{k-1}\right\|^{2} \\
& \leq\left\|u^{k}-u_{*}\right\|^{2}+2 \theta_{k}\left\|u^{k}-u_{*}\right\|\left\|u^{k}-u^{k-1}\right\|+\theta_{k}^{2}\left\|u^{k}-u^{k-1}\right\|^{2} .
\end{aligned}
$$

By (15)-(17), we see that

$$
\begin{aligned}
\left\|u^{k+1}-u_{*}\right\|^{2} \leq & \left\|u^{k}-u_{*}\right\|^{2}+2 \theta_{k}\left\|u^{k}-u_{*}\right\|\left\|u^{k}-u^{k-1}\right\|+\theta_{k}^{2}\left\|u^{k}-u^{k-1}\right\|^{2} \\
& -2 \alpha_{k}\left[(f+g)\left(v^{k}\right)-(f+g)\left(u_{*}\right)\right]-(1-2 \delta)\left\|w^{k}-v^{k}\right\|^{2} \\
& -\left\|P_{\Omega}\left(v^{k}\right)-v^{k}\right\|^{2} .
\end{aligned}
$$

This gives

$$
\begin{aligned}
\left\|P_{\Omega}\left(v^{k}\right)-v^{k}\right\|^{2}+(1-2 \delta)\left\|w^{k}-v^{k}\right\|^{2} \leq & \left(\left\|u^{k}-u_{*}\right\|^{2}-\left\|u^{k+1}-u_{*}\right\|^{2}\right) \\
& +2 \theta_{k}\left\|u^{k}-u_{*}\right\|\left\|u^{k}-u^{k-1}\right\| \\
& +\theta_{k}^{2}\left\|u^{k}-u^{k-1}\right\|^{2} .
\end{aligned}
$$

Since $\sum_{k=1}^{\infty} \theta_{k}\left\|u^{k}-u^{k-1}\right\|<+\infty$ and $\lim _{k \rightarrow \infty}\left\|u^{k}-u_{*}\right\|$ exists, it follows that $\left\|v^{k}-w^{k}\right\| \rightarrow 0$ and $\left\|u^{k+1}-v^{k}\right\| \rightarrow 0$. It is easily seen that $\left\|w^{k}-u^{k}\right\| \rightarrow 0$ and hence $\left\|u^{k+1}-u^{k}\right\| \rightarrow 0$. On the other hand, we see that

$$
\begin{aligned}
\left\|u^{k}-u_{*}\right\|^{2}-\left\|u^{k+1}-u_{*}\right\|^{2} \geq & 2 \alpha_{k}\left[(f+g)\left(v^{k}\right)-(f+g)\left(u_{*}\right)\right]+(1-2 \delta)\left\|w^{k}-v^{k}\right\|^{2} \\
& +\left\|P_{\Omega}\left(v^{k}\right)-u_{*}\right\|^{2} \\
\geq & (1-2 \delta)\left\|w^{k}-v^{k}\right\|^{2}+\left\|P_{\Omega}\left(v^{k}\right)-u_{*}\right\|^{2} \\
\geq & 0 .
\end{aligned}
$$

It follows that $\left(u^{k}\right)$ is Fejér convergent to $S_{*}$. Thus, we have

$$
\begin{aligned}
0 & \leq 2 \alpha_{k}\left[(f+g)\left(v^{k}\right)-(f+g)\left(u_{*}\right)\right] \\
& \leq\left\|u^{k}-u_{*}\right\|^{2}-\left\|u^{k+1}-u_{*}\right\|^{2} \\
& =\left(\left\|u^{k}-u_{*}\right\|-\left\|u^{k+1}-u_{*}\right\|\right)\left(\left\|u^{k}-u_{*}\right\|+\left\|u^{k+1}-u_{*}\right\|\right) \\
& \leq 2 M\left(\left\|u^{k}-u_{*}\right\|-\left\|u^{k+1}-u_{*}\right\|\right) \\
& \leq 2 M\left\|u^{k+1}-u^{k}\right\|,
\end{aligned}
$$

where $M=\sup \left\{\left\|u^{k}-u_{*}\right\| \mid k \in \mathbb{N}\right\}<+\infty$. Since $\left(u^{k}\right)$ is bounded, the set of weak accumulation points is nonempty. Take any weak accumulation point $\bar{u}$ of $\left(u^{k}\right)$. Thus, there is a subsequence $\left(u^{k_{n}}\right)$ of $\left(u^{k}\right)$ weakly converging to $\bar{u}$. Moreover, $\left\{w^{k_{n}}\right\}$ also weakly converges to $\bar{u}$. Since $\left(u^{k_{n}}\right)$ is bounded and $\left\|w^{k_{n}}-v^{k_{n}}\right\| \rightarrow 0$, from (A2), we obtain

$$
\lim _{n \rightarrow \infty}\left\|\nabla f\left(w^{k_{n}}\right)-\nabla f\left(v^{k_{n}}\right)\right\|=0 .
$$

Since $v^{k_{n}}=\operatorname{prox}_{\alpha_{k_{n} g} g}\left(w^{k_{n}}-\alpha_{k_{n}} \nabla f\left(w^{k_{n}}\right)\right)$, it follows from (7) that

$$
\frac{w^{k_{n}}-\alpha_{k_{n}} \nabla f\left(w^{k_{n}}\right)-v^{k_{n}}}{\alpha_{k_{n}}} \in \partial g\left(v^{k_{n}}\right),
$$

which yields 


$$
\frac{w^{k_{n}}-v^{k_{n}}}{\alpha_{k_{n}}}+\nabla f\left(w^{k_{n}}\right)-\nabla f\left(v^{k_{n}}\right) \in \nabla f\left(v^{k_{n}}\right)+\partial g\left(v^{k_{n}}\right) \in \partial(f+g)\left(v^{k_{n}}\right) .
$$

By passing $n \rightarrow \infty$ in (19), we get from (18) and Lemma 2 that $0 \in \partial(f+g)(\bar{u})$. Thus, $\bar{u} \in S_{*}$. By Lemma 5 (ii), we conclude that $\left(u^{k}\right)$ weakly converges to a point in $S_{*}$.

\section{Numerical Experiments}

In this section, we aim to apply our result for solving an image inpainting problem which is of the following mathematical model:

$$
\min _{u \in \mathbb{R}^{M \times N}} \frac{1}{2}\left\|A\left(u-u^{0}\right)\right\|_{F}^{2}+u\|u\|_{*}
$$

where $u^{0} \in \mathbb{R}^{M \times N}(M<N), A$ is a linear map that selects a subset of the entries of an $M \times N$ matrix by setting each unknown entry in the matrix to $0, u$ is matrix of known entries $A\left(u^{0}\right)$, and $\mu>0$ is regularization parameter.

In particular, we investigate the image inpainting problem $[25,26]$ :

$$
\left.\min _{u \in \mathbb{R}^{M \times N}} \frac{1}{2} \| P_{\Omega}(u)-P_{\Omega}\left(u^{0}\right)\right)\left\|_{F}^{2}+\mu\right\| u \|_{*}
$$

where $\|\cdot\|_{F}$ is the Frobenius matrix norm, and $\|\cdot\|_{*}$ is the nuclear matrix norm. Here, we define $P_{\Omega}$ as follows:

$$
P_{\Omega}(u)= \begin{cases}u_{i j}, & (i, j) \in \Omega, \\ 0, & \text { otherwise }\end{cases}
$$

The optimization problem (21) relates to (4). In fact, let $f(u)=\frac{1}{2}\left\|P_{\Omega}(u)-P_{\Omega}\left(u^{0}\right)\right\|_{F}^{2}$ and $g(u)=\mu\|u\|_{*}$. Then, $\nabla f(u)=P_{\Omega}(u)-P_{\Omega}\left(u^{0}\right)$ is 1-Lipschitz continuous. Moreover, prox $_{g}$ is obtained by the singular value decomposition (SVD) [27].

From Algorithm 5, we obtain the Algorithm 6 for image inpainting.

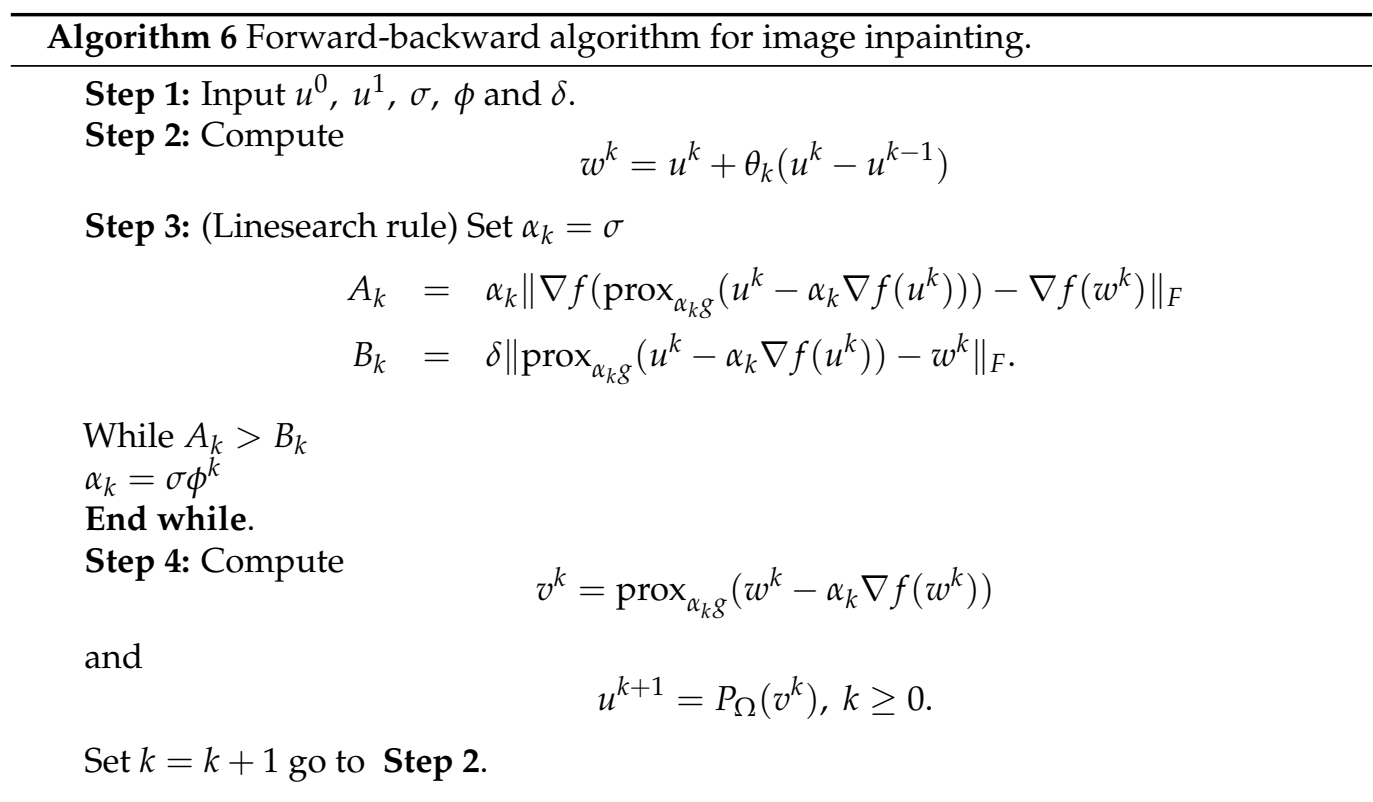

To measure the quality of images, we consider the signal-to-noise ratio (SNR) and the structural similarity index (SSIM) [28], which are given by

$$
\mathrm{SNR}=20 \log \frac{\|u\|_{F}}{\left\|u-u^{r}\right\|_{F}}
$$


and

$$
\operatorname{SSIM}=\frac{\left(2 a_{u} a_{u^{r}}+c_{1}\right)\left(2 \sigma_{u u^{r}}+c_{2}\right)}{\left(a_{u}^{2}+a_{u^{r}}^{2}+c_{1}\right)\left(\sigma_{u}^{2}+\sigma_{u^{r}}^{2}+c_{2}\right)}
$$

where $u$ is the original image, $u^{r}$ is the restored image, $a^{u}$ and $a_{u^{r}}$ are the mean values of the original image $u$ and restored image $u^{r}$, respectively, $\sigma_{u}^{2}$ and $\sigma_{u^{r}}^{2}$ are the variances, $\sigma_{u u^{r}}^{2}$ is the covariance of two images, $c_{1}=(0.01 L)^{2}$ and $c_{2}=(0.03 L)^{2}$, and $L$ is the dynamic range of pixel values. SSIM ranges from 0 to 1 , and 1 means perfect recovery. Next, we analyze its convergence including its effects of the parameters $\delta, \phi$ and $\sigma$ that proposed in Algorithm 5. We now present the corresponding numerical results (number of iterations denoted by Iter and CPU denoted by the time of CPU).

First, we investigate the effect $\delta$. Set parameters as follows:

$$
\theta_{k}= \begin{cases}\frac{t_{k}-1}{t_{k+1}} & \text { if } 1 \leq k<N, \\ \frac{1}{2^{k}} & \text { otherwise, }\end{cases}
$$

where $t_{k}$ is a sequence defined by $t_{1}=1$ and $t_{k+1}=\frac{1+\sqrt{1+4 t_{k}^{2}}}{2}$.

From Table 1, we observe that SNR and SSIM of Algorithm 5 have been getting larger when the parameter $\delta$ approaches 0.5. Moreover, the CPU of Algorithm 5 is decreasing when $\delta$ tends to 0.5 .

\begin{tabular}{|c|c|c|c|c|c|c|}
\hline \multicolumn{7}{|c|}{ Set: $\sigma=1$ and $\phi=0.4$} \\
\hline \multirow[t]{2}{*}{$\delta$} & \multicolumn{3}{|c|}{$\begin{array}{c}N=227, M=340 \\
\text { Iter }=60\end{array}$} & \multicolumn{3}{|c|}{$\begin{array}{c}N=480, M=360 \\
\text { Iter }=90\end{array}$} \\
\hline & SNR & SSIM & CPU & SNR & SSIM & CPU \\
\hline 0.5 & 22.8626 & 0.9476 & 2.2656 & 26.3994 & 0.9210 & 11.5229 \\
\hline 0.1 & 21.8228 & 0.9437 & 4.0916 & 26.3982 & 0.9209 & 21.7439 \\
\hline 0.05 & 14.7249 & 0.9165 & 5.0465 & 26.3974 & 0.9181 & 27.2411 \\
\hline 0.01 & 9.9919 & 0.8886 & 6.7001 & 19.2859 & 0.8939 & 37.8360 \\
\hline 0.001 & -0.7012 & 0.3889 & 8.0271 & 5.9239 & 0.6228 & 47.7375 \\
\hline
\end{tabular}

Table 1. The convergence results of Algorithm 5 for each $\delta$.

Figures 1-4 show the SNR and the reconstructed images for each caseof dimensions.

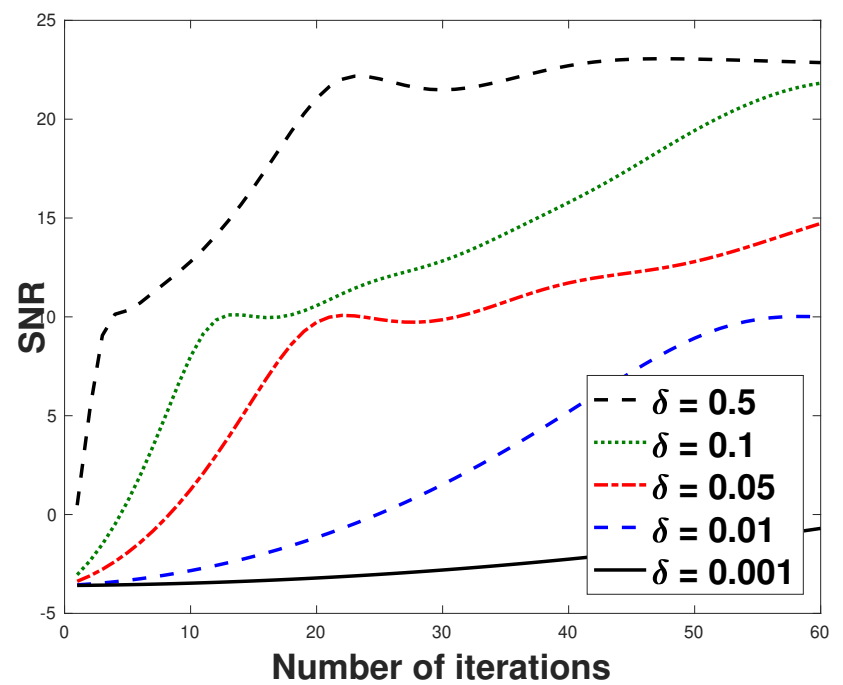

(a)

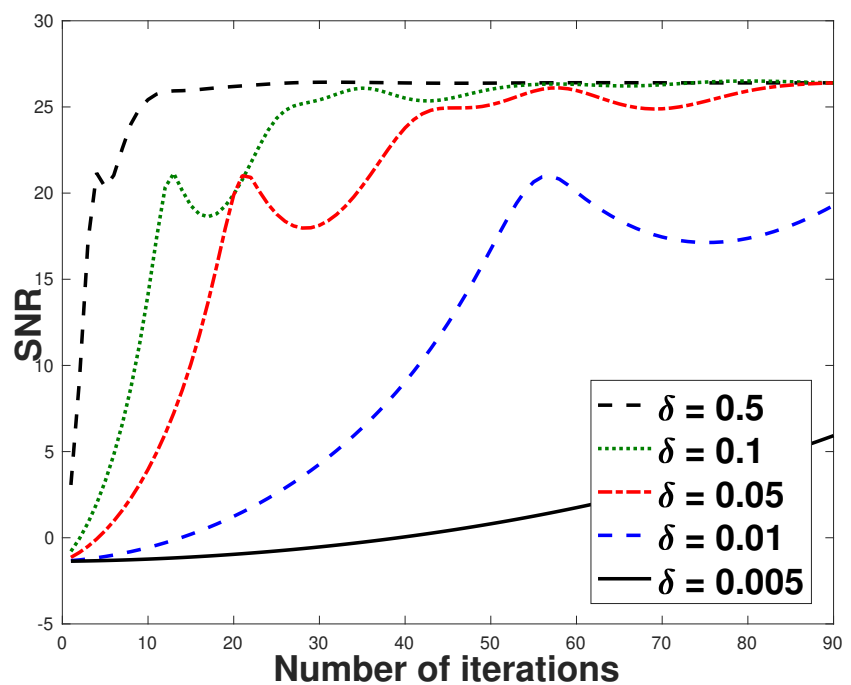

(b)

Figure 1. Graph of the number of iterations and SNR for the parameter $\delta$. (a) Number of iterations and SNR with $N=227$, $M=340$; (b) number of iterations and SNR with $N=480, M=360$. 


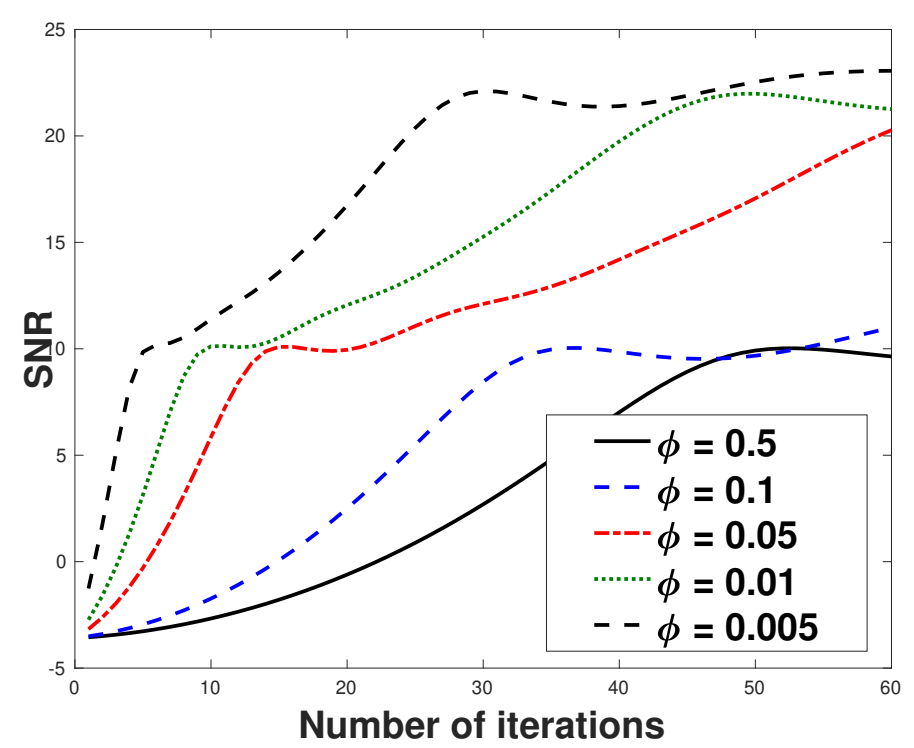

(a)

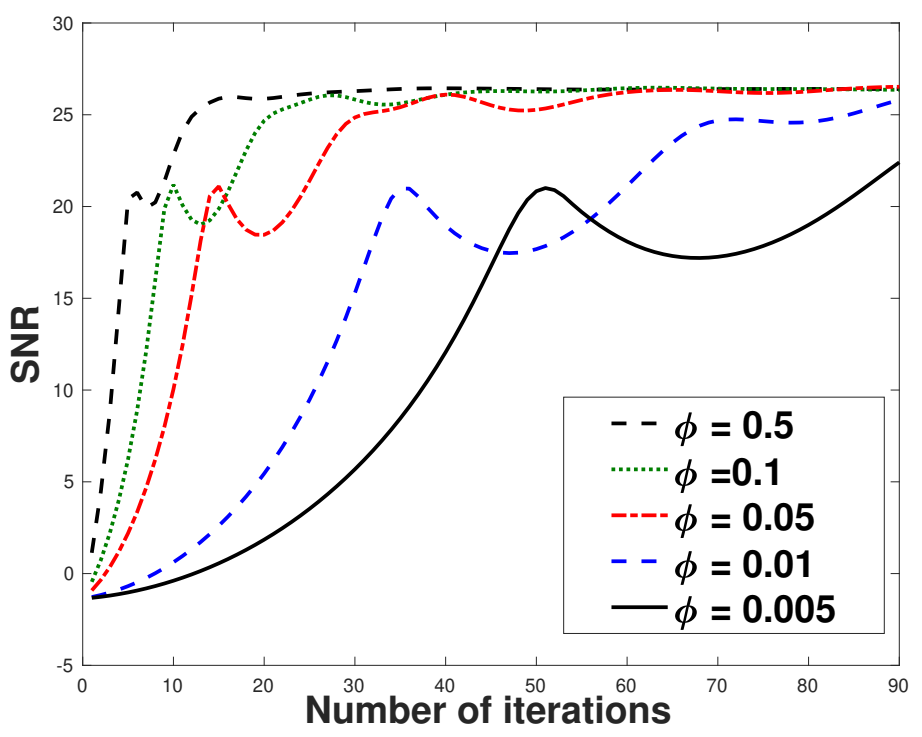

(b)

Figure 2. Graph of number of iterations and SNR for the parameter $\phi$. (a) Number of iterations and SNR with $N=227$, $M=340$; (b) number of iterations and SNR with $N=480, M=360$.

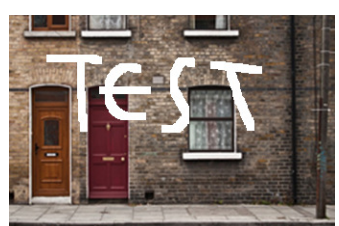

(a)

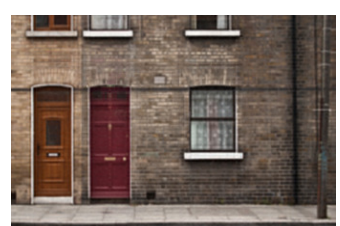

(b)

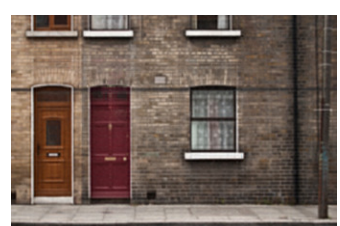

(c)

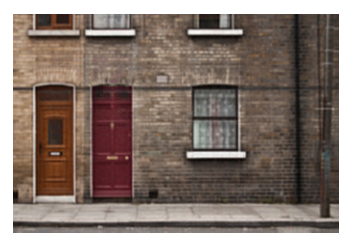

(d)

Figure 3. The painted image and restored images with the real image $N=227, M=340$. (a) The painted image; $(\mathbf{b})$ restored images in Table 1 for $\delta=0.5(\mathrm{SNR}=22.8626, \mathrm{SSIM}=0.9476)$; (c) restored images in Table 2 for $\phi=0.5(\mathrm{SNR}=23.0594$, SSIM $=0.9479) ;(\mathbf{d})$ restored images in Table 3 for $\sigma=5$ $(\mathrm{SNR}=22.9865, \mathrm{SSIM}=0.9477)$.

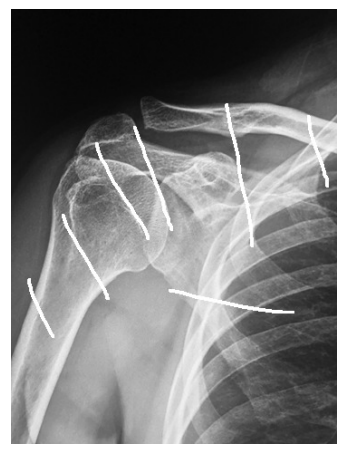

(a)

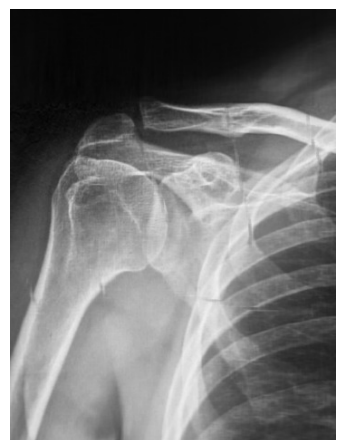

(b)

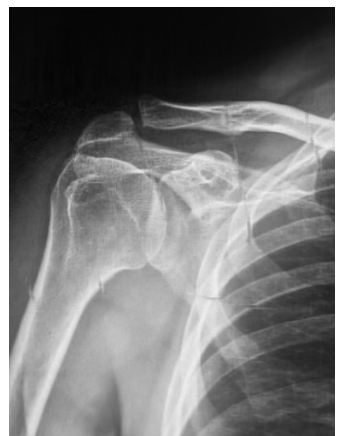

(c)

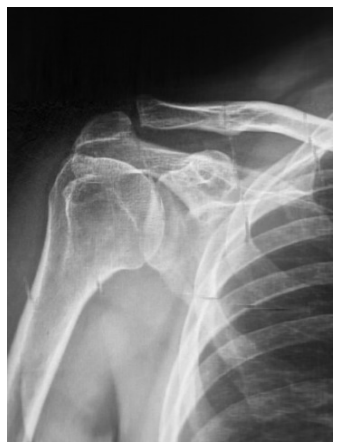

(d)

Figure 4. The painted image and restored images with the real image $N=480, M=360$. (a) The painted image; $(\mathbf{b})$ restored images in Table 1 for $\delta=0.5(\mathrm{SNR}=26.3994, \mathrm{SSIM}=0.9210)$; (c) restored images in Table 2 for $\phi=0.5(\mathrm{SNR}=26.4002$, SSIM =0.9210); $(\mathbf{d})$ restored images in Table 3 for $\sigma=0.5(\mathrm{SNR}=26.4084, \mathrm{SSIM}=0.9210)$.

Next, we discuss the effect of $\phi$. The numerical experiments are given in Table 2. 
Table 2. The convergence results of Algorithm 5 for each $\phi$.

\begin{tabular}{ccccccc}
\hline \multicolumn{7}{c}{ Given: $\delta=\mathbf{0 . 4}$ and $\sigma=\mathbf{1}$} \\
\hline \multirow{2}{*}{$\boldsymbol{\phi}$} & \multicolumn{3}{c}{$N=\mathbf{2 2 7}, \mathbf{M = 3 4 0}$} & \multicolumn{3}{c}{ Iter $=\mathbf{9 0}$} \\
\cline { 2 - 7 } & \multicolumn{3}{c}{ Iter $=\mathbf{6 0}$} & $\mathbf{4 8 0 ,} \boldsymbol{M}=\mathbf{3 6 0}$ \\
\cline { 2 - 7 } & SNR & SSIM & CPU & SNR & SSIM & CPU \\
\hline 0.5 & 23.0594 & 0.9479 & 3.0865 & 26.4002 & 0.9210 & 17.0693 \\
0.1 & 21.2594 & 0.9428 & 2.2104 & 26.3604 & 0.9205 & 11.4325 \\
0.05 & 20.2735 & 0.9391 & 2.1874 & 26.5203 & 0.9207 & 11.5494 \\
0.01 & 10.9957 & 0.8918 & 2.2586 & 25.8063 & 0.9114 & 11.2883 \\
0.005 & 9.6387 & 0.8658 & 2.2449 & 22.4065 & 0.9095 & 11.3964 \\
\hline
\end{tabular}

From Table 2, we observe that SNR, SSIM, and CPU time of Algorithm 5 have been getting larger when the parameter $\phi$ approaches 0.5 .

Next, we discuss the effect $\sigma$. The numerical experiments are given in Table 3. From Table 3, we observe that SNR, SSIM, and the CPU time of Algorithm 5 have been getting larger if $\sigma$ increases. The SNR and the reconstructed images are shown in Figures 3-5.

Table 3. The convergence results of Algorithm 5 for each $\sigma$.

\begin{tabular}{ccccccc}
\hline \multicolumn{7}{c}{ Set: $\delta=\mathbf{0 . 4}$ and $\boldsymbol{\phi}=\mathbf{0 . 4}$} \\
\hline$\sigma$ & \multicolumn{3}{c}{$\begin{array}{c}N=\mathbf{2 2 7}, \boldsymbol{M}=\mathbf{3 4 0} \\
\text { Iter }=\mathbf{6 0}\end{array}$} & & $\begin{array}{c}N=\mathbf{4 8 0 ,} \boldsymbol{M}=\mathbf{3 6 0} \\
\text { Iter }=\mathbf{9 0}\end{array}$ \\
\cline { 2 - 7 } & SNR & SSIM & CPU & SNR & SSIM & CPU \\
\hline 5 & 22.9865 & 0.9477 & 3.9889 & 26.3946 & 0.9209 & 21.2420 \\
1 & 22.8626 & 0.9476 & 2.2848 & 26.3994 & 0.9210 & 11.1571 \\
0.5 & 22.8327 & 0.9474 & 2.3458 & 26.4084 & 0.9210 & 11.2728 \\
0.05 & 20.2735 & 0.9391 & 1.3531 & 26.5230 & 0.9207 & 6.0226 \\
0.005 & 9.6387 & 0.8638 & 1.3434 & 22.4065 & 0.9095 & 6.2738 \\
\hline
\end{tabular}

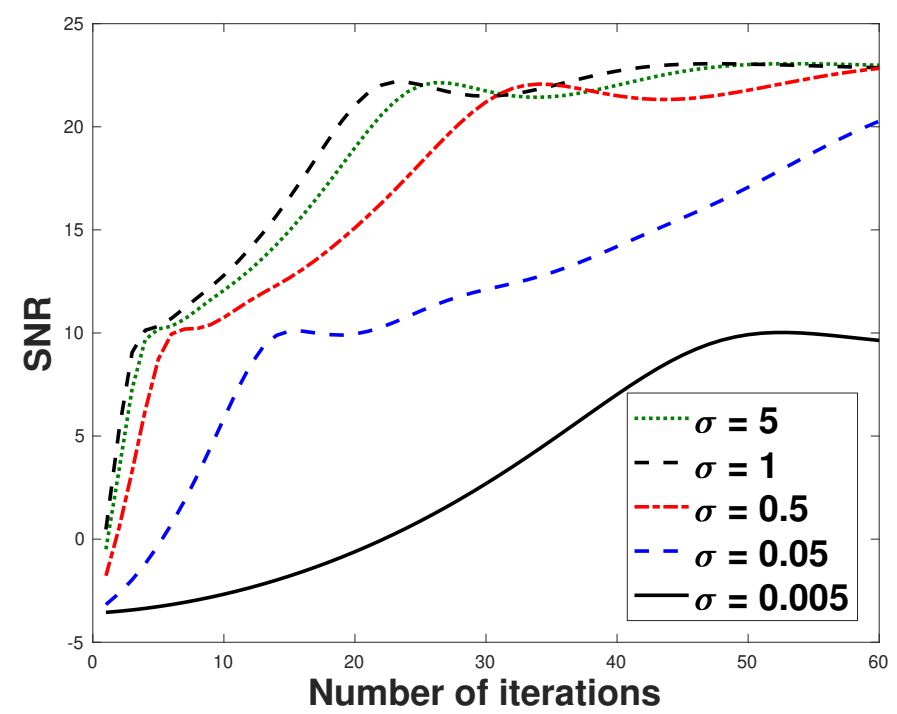

(a)

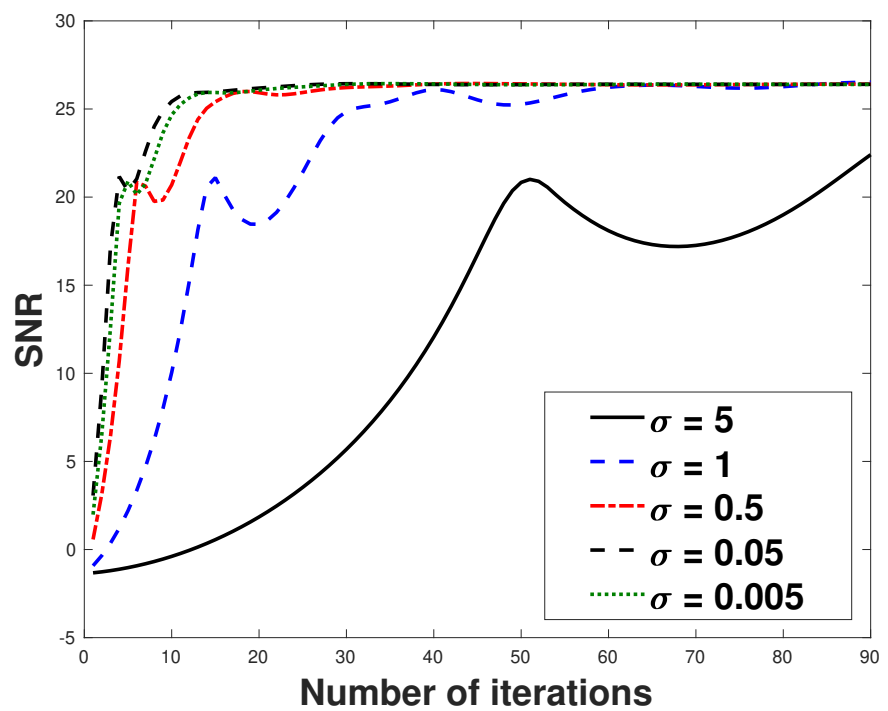

(b)

Figure 5. Graph of the number of iterations and SNR for the parameter $\sigma$. (a) Number of iterations and SNR with $N=227$, $M=340$; (b) number of iterations and SNR with $N=480, M=360$.

The real images are shown in Figure 6, input image, and the reconstructed images are shown in Figures 3, 4 and 6, respectively. 


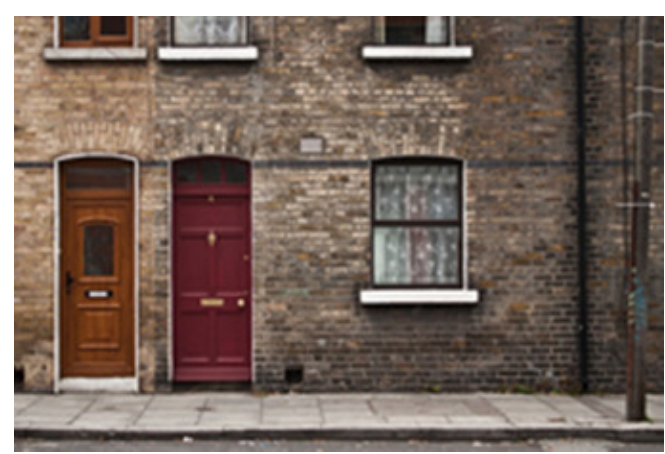

(a)

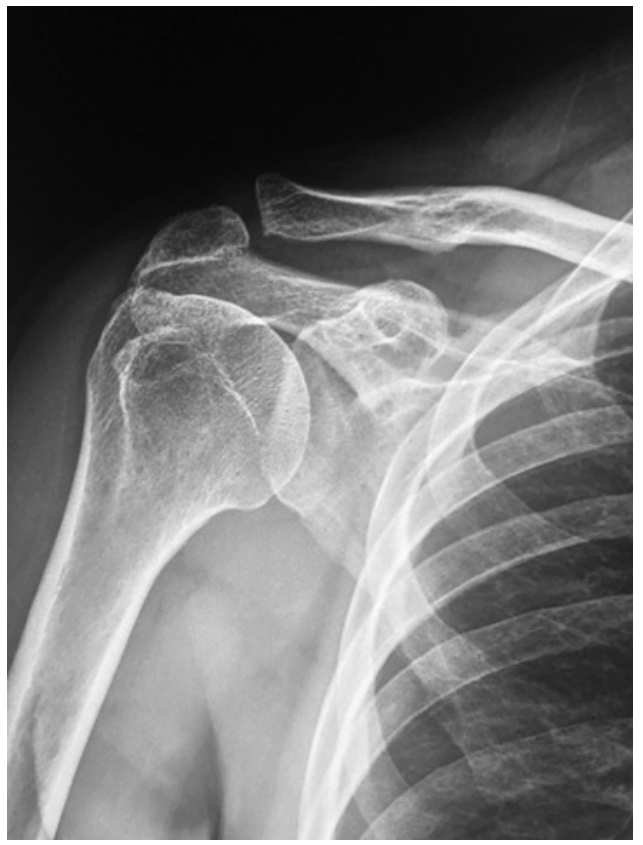

(b)

Figure 6. The original images. (a) The original image of size $N=227, M=340$; (b) the original image of size of $N=480, M=360$.

Now, we present the performance of Algorithms 5 and its comparison to the projected version of Algorithm 1 [13] and Algorithm 2 [14]. The initial point $u^{0}$ and $u^{1}$ are chosen to be zero and let $\alpha_{k}=\frac{1}{\|A\|^{2}}$ and $\lambda_{k}=0.09$ in Algorithm 1. Let $\sigma=0.1, \delta=0.13, \phi=0.5$ and $\theta$ be defined by (25) in Algorithms 2 and 5, respectively. The numerical results are shown in Table 4.

Table 4. Computational results for solving (21).

\begin{tabular}{lcccc}
\hline & \multicolumn{2}{c}{ Image Size } & \multicolumn{2}{c}{ Image Size } \\
\cline { 2 - 5 } & SNR & SSIM & SNR & SSIM \\
\hline Algorithm 1 & 19.8276 & 0.9378 & 25.7210 & 0.9363 \\
Algorithm 2 & 20.4704 & 0.9402 & 26.2362 & 0.9373 \\
Algorithm 5 & 22.9158 & 0.9477 & 27.6581 & 0.9400 \\
\hline
\end{tabular}

From Table 4, we see that the experiment results of Algorithm 5 are better than Algorithms 1 and 2 in terms of SNR and SSIM in all cases.

The figure of the inpainting image for the 260th and 310th iterations are shown in Figures 7-9, respectively.

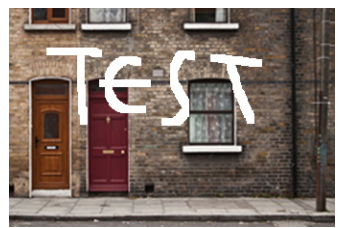

(a)

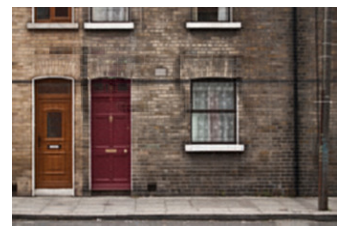

(b)

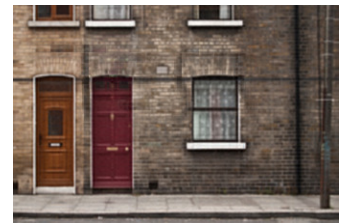

(c)

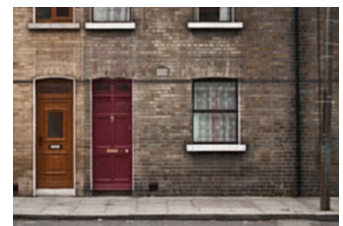

(d)

Figure 7. The painted image and restored images. (a) The painted image; (b) restored images in Algorithm 1 (SNR = 19.8276, SSIM = 0.9278); (c) restored images in Algorithm 2 (SNR = 20.4704, SSIM $=0.9402) ;(d)$ restored images in Algorithm $5($ SNR $=22.9158$, SSIM $=0.9477)$. 


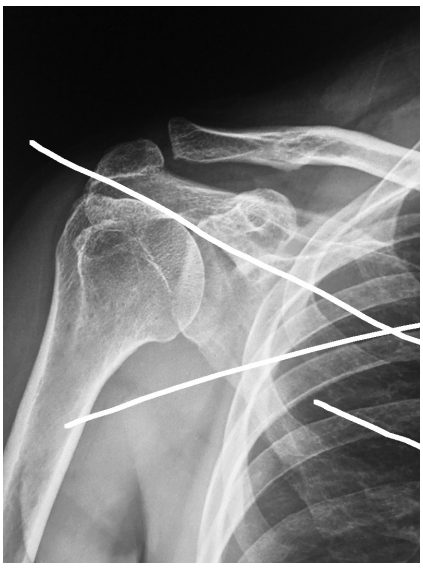

(a)

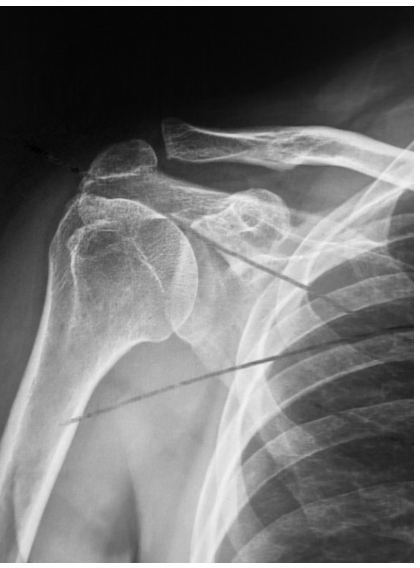

(b)

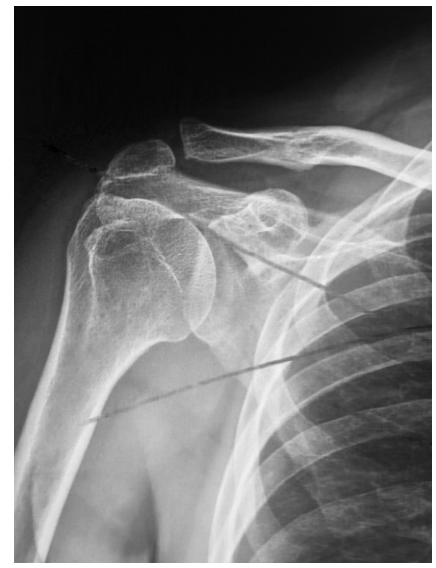

(c)

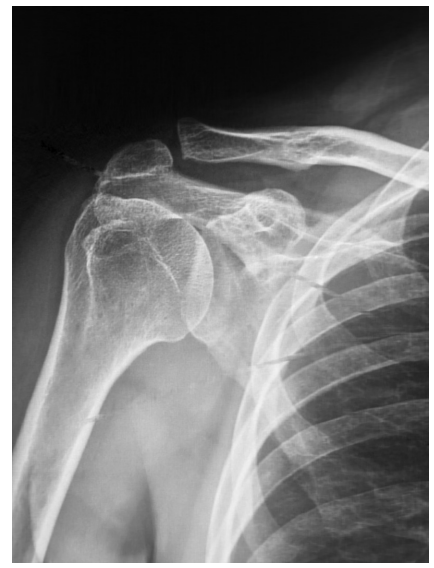

(d)

Figure 8. The painted image and restored images. (a) The painted image; (b) restored images in Algorithm 1 (SNR $=25.7210$, SSIM = 0.9363); (c) restored images in Algorithm 2 (SNR = 26.2362, SSIM = 0.9373); (d) restored images in Algorithm 5 $(\mathrm{SNR}=27.6581, \mathrm{SSIM}=0.9400)$.

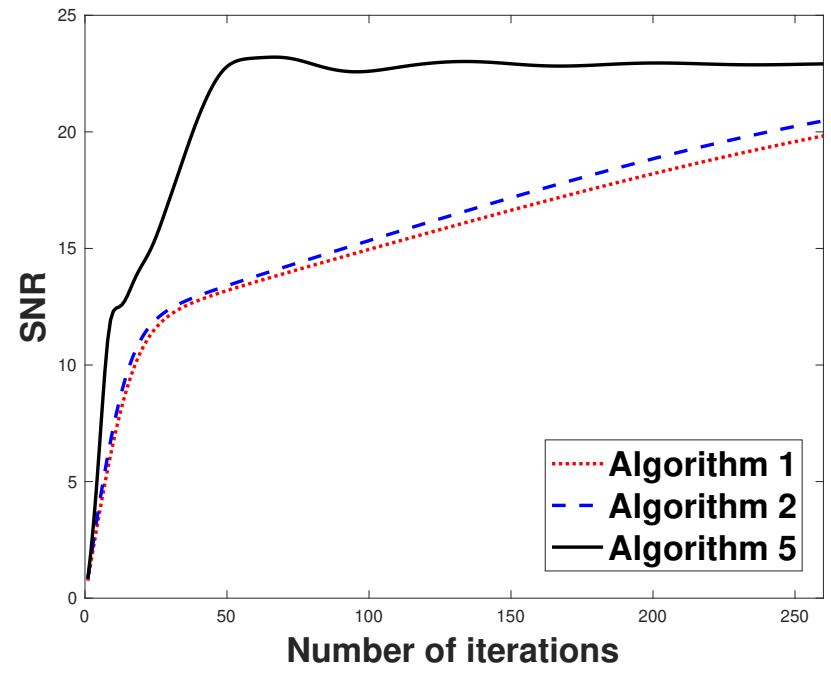

(a)

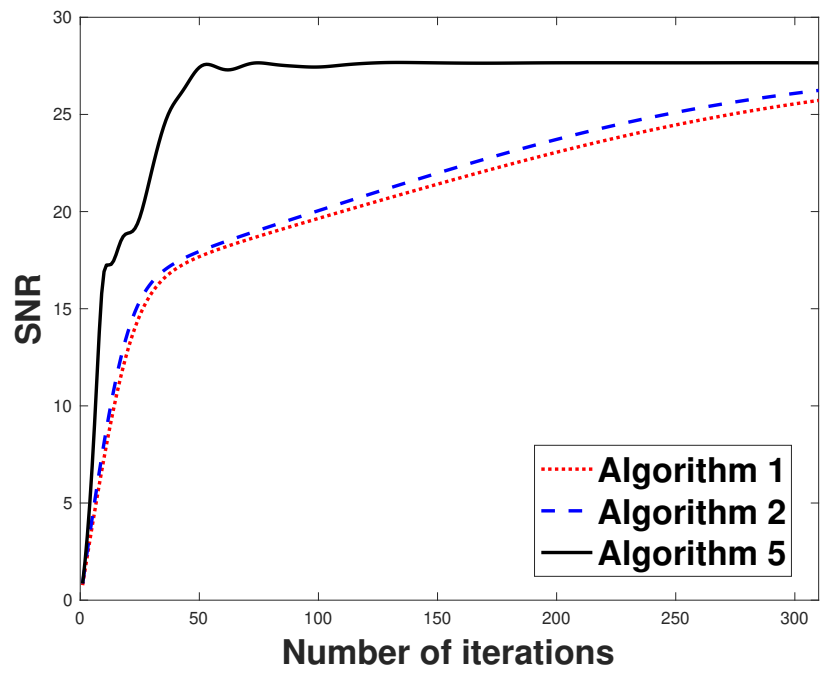

(b)

Figure 9. The SNR value and number of iterations for all cases. (a) Graph of SNR value and number of iterations of Figure 7; (b) graph of SNR value and number of iterations of Figure 8.

\section{Conclusions}

In this research, we investigated inertial projected forward-backward algorithm using linesearches for constrained minimization problems. The weak convergence results were proved under control conditions. The proposed algorithms do not need to compute the Lipschitz constant of the gradient of functions. We applied our results to solve image inpainting. We also presented the effects of all parameters that are assumed in our method.

For our future research, we aim to find a new linesearch technique that does not require the Lipschitz continuity assumption on the gradient of the function. We note that the proposed algorithm depends on the computation of the projection which is not an easy task to find in some cases. It is interesting to construct new algorithms that do not involve the projection.

Author Contributions: Funding acquisition and supervision, S.S.; writing-original draft preparation, K.K.; writing-review and editing and software, P.C. All authors have read and agreed to the published version of the manuscript. 
Funding: This research was funded by Chiang Mai University and Thailand Science Research and Innovation under the project IRN62W007.

Institutional Review Board Statement: Not applicable.

Informed Consent Statement: Not applicable.

Data Availability Statement: Not applicable.

Acknowledgments: The authors would like to thank reviewers and the editor for valuable comments for improving the original manuscript.

Conflicts of Interest: The authors declare no conflict of interest.

\section{References}

1. Bauschke, H.H.; Borwein, J.M. Dykstra's alternating projection algorithm for two sets. J. Approx. Theory 1994, 79, 418-443. [CrossRef]

2. Chen, G.H.; Rockafellar, R.T. Convergence rates in forward-backward splitting. SIAM J. Optim. 1997, 7, 421-444. [CrossRef]

3. Cholamjiak, W.; Cholamjiak, P.; Suantai, S. An inertial forward-backward splitting method for solving inclusion problems in Hilbert spaces. J. Fixed Point Theory Appl. 2018, 20, 1-7. [CrossRef]

4. Dong, Q.; Jiang, D.; Cholamjiak, P.; Shehu, Y. A strong convergence result involving an inertial forward-backward algorithm for monotone inclusions. J. Fixed Point Theory Appl. 2017, 19, 3097-3118. [CrossRef]

5. Bauschke, H.H.; Burachik, R.S.; Combettes, P.L.; Elser, V.; Luke, D.R.; Wolkowicz, H. (Eds.) Fixed-Point Algorithms for Inverse Problems in Science and Engineering; Springer: Berlin/Heidelberg, Germany, 2011.

6. Kankam, K.; Pholasa, N.; Cholamjiak, P. Hybrid Forward-Backward Algorithms Using Linesearch Rule for Minimization Problem. Thai J. Math. 2019, 17, 607-625.

7. Dunn, J.C. Convexity, monotonicity, and gradient processes in Hilbert space. J. Math. Anal. Appl. 1976, 53, 145-158. [CrossRef]

8. Wang, C.; Xiu, N. Convergence of the gradient projection method for generalized convex minimization. Comput. Optim. Appl. 2000, 16, 111-120. [CrossRef]

9. Xu, H.K. Averaged mappings and the gradient-projection algorithm. J. Optim. Theory Appl. 2011, 150, 360-378. [CrossRef]

10. Güler, O. On the convergence of the proximal point algorithm for convex minimization. SIAM J. Control Optim. 1991, 29, 403-419. [CrossRef]

11. Martinet, B. Régularisation dinéquations variationnelles par approximations successives. Rev. Franaise Informat. Rech. OpéRationnelle 1970, 4, 154-158.

12. Rockafellar, R.T. Monotone operators and the proximal point algorithm. SIAM J. Control Optim. 1976, 14, 877-898. [CrossRef]

13. Combettes, P.L.; Wajs, V.R. Signal recovery by proximal forward-backward splitting. Multiscale Model. Simul. 2005, 4, 1168-1200. [CrossRef]

14. Bello Cruz, J.Y.; Nghia, T.T. On the convergence of the forward-Backward splitting method with linesearches. Optim. Methods Softw. 2016, 31, 1209-1238. [CrossRef]

15. Polyak, B.T. Some methods of speeding up the convergence of iteration methods. Ussr Comput. Math. Math. Phys. 1964, 4, 1-7. [CrossRef]

16. Nesterov, Y.E. A method for solving the convex programming problem with convergence rate $O\left(1 / k^{2}\right)$. InDokl. Akad. Nauk Sssr 1983, 269, 543-547.

17. Moudafi, A.; Oliny, M. Convergence of a splitting inertial proximal method for monotone operators. J. Comput. Appl. Math. 2003, 155, 447-454. [CrossRef]

18. Beck, A.; Teboulle, M. A fast iterative shrinkage-thresholding algorithm for linear inverse problems. SIAM J. Imaging Sci. 2009, 2, 183-202. [CrossRef]

19. Suantai, S.; Pholasa, N.; Cholamjiak, P. The modified inertial relaxed CQ algorithm for solving the split feasibility problems. J. Ind. Manag. Optim. 2018, 14, 1595. [CrossRef]

20. Shehu, Y.; Cholamjiak, P. Iterative method with inertial for variational inequalities in Hilbert spaces. Calcolo 2019, 56, 1-21. [CrossRef]

21. Bauschke, H.H.; Combettes, P.L. Convex Analysis and Monotone Operator Theory in Hilbert Spaces; Springer: New York, NY, USA, 2011; Volume 408.

22. Burachik, R.S.; Iusem, A.N. Enlargements of Monotone Operators. In InSet-Valued Mappings and Enlargements of Monotone Operators; Springer: Boston, MA, USA, 2008; pp. 161-220.

23. Hanjing, A.; Suantai, S. A fast image restoration algorithm based on a fixed point and optimization method. Mathematics 2020, 8, 378. [CrossRef]

24. Iusem, A.N.; Svaiter, B.F.; Teboulle, M. Entropy-like proximal methods in convex programming. Math. Oper. Res. 1994, 19, 790-814. [CrossRef]

25. Cui, F.; Tang, Y.; Yang, Y. An inertial three-operator splitting algorithm with applications to image inpainting. arXiv 1904, arXiv:1904.11684. 
26. Davis, D.; Yin, W. A three-operator splitting scheme and its optimization applications. Set-Valued Var. Anal. 2017, $25,829-858$. [CrossRef]

27. Cai, J.F.; Candès, E.J.; Shen, Z. A singular value thresholding algorithm for matrix completion. SIAM J. Optim. 2010, 20, 1956-1982. [CrossRef]

28. Wang, Z.; Bovik, A.C.; Sheikh, H.R.; Simoncelli, E.P. Image quality assessment: from error visibility to structural similarity. IEEE Trans. Image Process. 2004, 13, 600-612. [CrossRef] 